\title{
Electric field strength on MHD aluminum alloys (AA7075) nanofluid flow
}

\author{
R. Kandasamy ${ }^{1} \cdot$ Nur Atikah bt Adnan ${ }^{1} \cdot$ Mohd Radiah $^{1} \cdot$ Mohd Kamarulzaki $^{1}$
}

(C) Springer Nature Switzerland AG 2018

\begin{abstract}
This work concerns with a numerical study on heat transfer by MHD aluminum alloys nanofluid (AA7075-water) flow over a nonlinear wall for extensible compactness of electric field. The steady governing momentum and energy equations are transformed to similarity equations for certain families of the controlling parameters. The transformed equations of momentum and thermal transport are solved by applying Runge-Kutta Fehlberg method with shooting technique. The three classical form of nanoparticle shapes are registered into report, i.e., sphere, cylinder and lamina and the heat transfer by natural convection of AA7075-water was studied. It is observed that the thermal and diffusive boundary layer thickness of AA7075-water is weaker in the presence of electric field, $E 1=0.2$ as compared to $E 1=0.0$. In the presence of electric field, temperature and concentration of lamina shape AA7075 particles hit a dominant role on AA7075-water nanofluid flow regime.
\end{abstract}

Keywords MHD AA7075-water - Wall thickness - Thermal radiation · Similarity solution · Viscous dissipation . Nanoparticle shapes

\section{Introduction}

Aluminum alloys are composed by melting, sintering (assemble of shaped parts utilizing metal particle that is melt together at exalted temperatures) or mechanical braiding. Durable collegial interruption to nanoparticles in distinct solvents is consistently entitled as "Nanofluids". In this analysis the distinct attitudes to nanofluids technology are defined with an insistence on utilization in energy organization. Aluminum alloys have been very influential in aerospace manufacturing. In special, the aluminum alloys such as AA7072 and AA7075 are very fruitful in transport utilizations such as marine, aviation and automotive which are also recycled in construction of bicycle equipment, glider rock climbing equipment and aircrafts. An aluminum alloy is a chemical design where new materials are combined with pure aluminum with regard to upgrading its resources, basically to enhance its stability. Alloy desires the complete associating of aluminum with these other materials although the aluminum is in liquidmolten-form. Superficial Aluminum alloy AA7075 was cultivated over Japanese in 1942, for construction of air frame in Japanese navy [1].

The boundary layer flow of an electrical controlling nanofluid against a stretching sheet has been an alive field of analysis freshly. Nanofluid is a fluid composed by a reliable interruption of micro/nano-sized particles (nonmetallic/metallic/nanofibers) with a classic size $<100 \mathrm{~nm}$ in a typical base fluid (liquid). One of these advances is the inclusion of small-sized particles to the liquid. This method contributes to raise the thermal conductivity of the base fluid relatively twice and the convective heat transfer achievement. The flow due to stretching boundary has inclusive analysis in the exclusion mechanisms associates in metal and plastic industries. An aluminum alloy is a synthetic configuration where modern materials are combined with ideal aluminum with employing to improve its resources, really to boost its solidity and

R. Kandasamy, ramasamy@uthm.edu.my| ${ }^{1}$ FAST, Universiti Tun Hussein Onn Malaysia, 84600 Pagoh, Johor, Malaysia.

SN Applied Sciences (2019) 1:12 | https://doi.org/10.1007/s42452-018-0014-y 
thermal conductivity. Aluminum alloys have been achieving a powerful issue in aerospace forming, [2-4]. Thermophoresis and Brownian motion effects on MHD bioconvection of nanofluid with nonlinear thermal radiation and quartic chemical reaction past an upper horizontal surface of a paraboloid of revolution with variable stream conditions are studied by [5-7].

Magnetohydrodynamic (MHD) nanofluid is an authentic significant in the field of engineering, science and technology. These fluids are more suitable in the optical grating, optical modulators, optical switches, tunable optical fiber filters, stretching of plastic materials, polymer industry and metallurgy. Nanotechnology investigation is repeatedly in the area of energetic scientific event due to an appreciable cast of useful utilizing in optical, medical and electrical fields and the nanoparticles thermal conductivity are more energetic as associated to the ideal fluids, as noticed in practical analysis was organized by [8-10]. The miserable of Lorentz force velocimetry is placed on appraisement of the Lorentz force that develops a convective fluid flow under the issue of an extensible magnetic field. Assigning to Faraday's law, when a metal or convective fluid efforts over a magnetic field, eddy currents expanded there by electromotive energy in zones of controlling magnetic field gradient. Eddy current in its support admits convinced magnetic field assigning to Ampère's law. The affiliation with eddy currents and total magnetic field allotments upgraded to Lorentz force that cracks the velocity and raises the temperature of the flow, $[11,12]$. Nonlinearity in the stretching sheet surface system has been consigned to exponential and quadratic (power-law) models, [13, 14].

The flow of electrically controlling fluids such as liquid metals is efficiently impressed by applied magnetic and electric fields. The issue has significant industrial utilizations in nuclear technology, metallurgy and other fields. The electric conductivity, which regulates the heat transit in an indirect aspect, is one of the well-designed of the transport assets because it is nearly easy to define. It has been well-defined that nanofluid flow, thus convective heat transfer, could be formed if the electrically controlling fluid is exposed to either electric or magnetic or combined fields. Electric conducting energy film nanofluid flow across a wall has been an energetic field of research freshly. This technique commits to advance conductivity of the common fluid about double and the convective heat transfer fulfillment [15-19]. Nanofluid placed magnetohydrodynamic hits a crucial role in technology, science and engineering [20-25]. Synthetic reaction impact is to estimate the reactor fulfillment of flexible stream conditions is investigated by [26-31]. Particle concentration depreciates for energetic Brownian motion although it rises for more dynamic thermophoretic of the nanofluid which rises the thermal attitude of the fluid [29-32]. A few experimental based articles have been investigated with aspect of hybrid nanofluids, [33-39].

The intent at this effort is delighted by the intimate survey and strong accessible industrial and engineering applications. The instant review introduces such distinct thickness on a nonlinear wall on electric MHD boundary layer AA7075-water flow in biography. This formable thickness nonlinear stretching sheet has energetic message on mechanical, civil, marine, aeronautical structure and designs. Confirmed conditions of the current work are analyzed and authorized graphically to real amplitudes convoluted within it and the prompt attainments are associated with the suitable literature.

\section{Mathematical formulation}

Investigate a steady electric MHD AA7075-water flow up a non-linear wall with flexible thickness. Wall velocity is designed as $U_{w}(x)=U_{0}(x+b)^{(n)}$, and the stretching sheet is appropriated at $y=A_{1}(x+b)^{\left(\frac{1-n}{2}\right)}$. The boundary layer equations are refined placed on Ohm's and Maxwell's laws in the existence of Electrical magnetohydrodynamics (EMHD). The incompressible AA7075-water viscous nanofluid with enhanced magnetic field $B$ and electric field $E$ is appropriated into consideration. The flow is in aspect of stretching to a sheet from a crack at two equal and opposite force and thermally radiative. The magnetic and electric zones acknowledge the Ohm's law define $\bar{J}=\sigma(\bar{E}+\bar{V} \times \bar{B})$ where $\bar{J}$-the Joule current, $\sigma$-the electrical conductivity and $\bar{V}$ - the fluid velocity. $E(x), B(x)$ -electric and magnetic strength is normal through the flow, such that the Reynolds number is endorsed small. The assured magnetic field is weaker to the confirmed magnetic field. So the assured magnetic field is insistent on small-scale magnetic Reynolds number. The twodimensional electrical MHD boundary layer flow equations of an incompressible electric conducting AA7075-water nanofluid are given as [19, 24, 37, 38] (Fig. 1)

$$
\begin{aligned}
& \frac{\partial v}{\partial y}=-\frac{\partial u}{\partial x} \begin{aligned}
u \frac{\partial u}{\partial x}+v \frac{\partial u}{\partial y}= & -\frac{1}{\rho_{n f}} \frac{\partial P}{\partial x}+\frac{\mu_{n f}}{\rho_{n f}}\left(\frac{\partial^{2} u}{\partial x^{2}}+\frac{\partial^{2} u}{\partial y^{2}}\right) \\
& -\frac{\sigma_{n f}}{\rho_{n f}}\left(B_{0}^{2}(x) u-E(x) B(x)\right)
\end{aligned} \\
& \begin{aligned}
\rho_{n f}\left(v \frac{\partial v}{\partial y}+u \frac{\partial v}{\partial x}\right)= & -\frac{\partial P}{\partial y}+\mu_{n f}\left(\frac{\partial^{2} v}{\partial x^{2}}+\frac{\partial^{2} v}{\partial y^{2}}\right) \\
& +\sigma_{n f}\left(E(x) B(x)-B_{0}^{2}(x) v\right)
\end{aligned}
\end{aligned}
$$




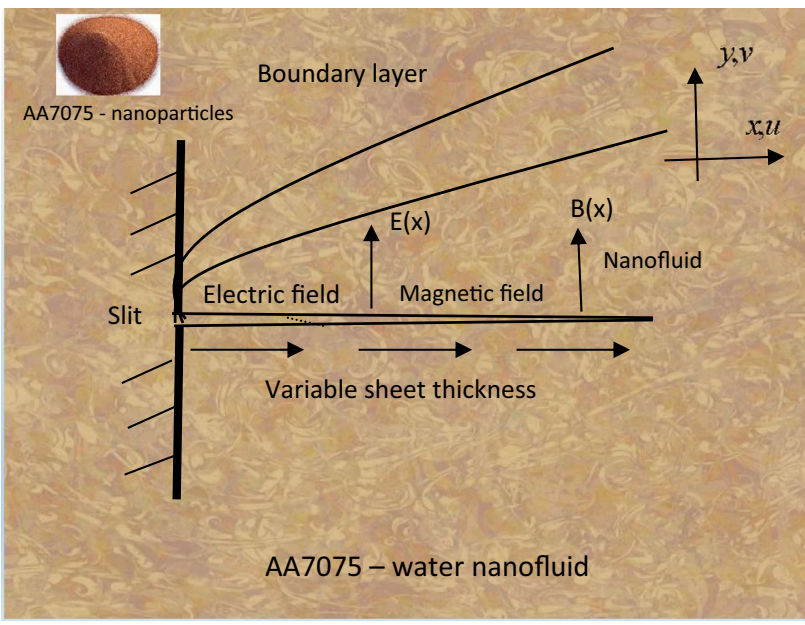

Fig. 1 Physical structure of flow geometry

$$
\begin{aligned}
u \frac{\partial T}{\partial x}+v \frac{\partial T}{\partial y}= & \frac{k_{n f}}{\left(\rho c_{p}\right)_{n f}}\left(\frac{\partial^{2} T}{\partial x^{2}}+\frac{\partial^{2} T}{\partial y^{2}}\right)+\frac{1}{\left(\rho c_{p}\right)_{n f}} \frac{\partial q_{r}}{\partial y} \\
& +\frac{\mu_{n f}}{\left(\rho c_{p}\right)_{n f}}\left(\frac{\partial u}{\partial y}\right)^{2}+\frac{\sigma_{n f}}{\rho_{n f}}(u B(x)-E(x))^{2} \\
& +\tau\left[\left(D_{B}\right)_{n f}\left\{\frac{\partial \phi}{\partial x} \frac{\partial T}{\partial x}+\frac{\partial \phi}{\partial y} \frac{\partial T}{\partial y}\right\}\right. \\
& \left.+\frac{\left(D_{T}\right)_{n f}}{T_{\infty}}\left\{\left(\frac{\partial T}{\partial x}\right)^{2}+\left(\frac{\partial T}{\partial y}\right)^{2}\right\}\right]
\end{aligned}
$$$$
\frac{\partial \phi}{\partial x}+v \frac{\partial \phi}{\partial y}=\left(D_{B}\right)_{n f}\left(\frac{\partial^{2} \phi}{\partial x^{2}}+\frac{\partial^{2} \phi}{\partial y^{2}}\right)
$$$$
+\frac{\left(D_{T}\right)_{n f}}{T_{\infty}}\left\{\frac{\partial^{2} T}{\partial x^{2}}+\frac{\partial^{2} T}{\partial y^{2}}\right\}-K^{*}\left(\phi-\phi_{\infty}\right)
$$

$u(x+b)^{-n}=U_{0}, v=0, T=T_{w^{\prime}} \phi=\phi_{w} y=A_{1}(x+b)^{\left(\frac{1-n}{2}\right)}$; $u \rightarrow 0, T \rightarrow T_{\infty}, \phi \rightarrow \phi_{\infty}$ as $y \rightarrow \infty$

Let $B(x)=B_{0}(x+b)^{\left(\frac{n-1}{2}\right)}$-magnetic field factor, $\sigma-$ electrical conductivity, $E(x)=E_{0}(x+b)^{\left(\frac{n-1}{2}\right)}$ _electric field factor, $c, b, n$-constants, $u, v$-velocity factor, $T$-nanofluid temperature, $\alpha_{n f}$-nanofluid thermal diffusivity, $\rho_{n f}$ —nanofluid effective density and $\left(\rho c_{p}\right)_{n f}$-nanofluid heat capacitance are explained [29] as:

$$
\begin{aligned}
\rho_{n f}-\zeta \rho_{s} & =(1-\zeta) \rho_{f}, \mu_{n f} \\
& =\frac{\mu_{f}}{(1-\zeta)^{2.5}},(\rho \beta)_{n f}-\zeta(\rho \beta)_{s}=(1-\zeta)(\rho \beta)_{f},\left(\rho c_{p}\right)_{n f} \\
& =(1-\zeta)\left(\rho c_{p}\right)_{f}+\zeta\left(\rho c_{p}\right)_{s} \\
\frac{\alpha_{n f}}{k_{n f}} & =\frac{1}{\left(\rho c_{p}\right)_{n f}}, \frac{k_{n f}}{k_{f}} \\
& =\left\{\frac{\left(k_{s}+(I-1) k_{f}\right)-(I-1) \zeta\left(k_{f}-k_{s}\right)}{\left(k_{s}+(I-1) k_{f}\right)+\zeta\left(k_{f}-k_{s}\right)}\right\}, \sigma_{n f}-\zeta \sigma_{s} \\
& =(1-\zeta) \sigma_{f}\left(D_{B}\right)_{n f}=(1-\zeta)\left(D_{B}\right)_{f}
\end{aligned}
$$

Maxwell design [40] was cultivated to assign the dynamic thermal or electrical conductivity of liquid-solid suspensions. Let $\sigma_{f}$ and $\sigma_{s}$-fluid and nanoparticle electrical conductivity, $k_{n f}$-nanofluid effective thermal conductivity. Rosseland's dissipation for the radiative heat flux, $q_{r}=-\frac{4 \sigma^{*} \partial T^{4}}{3 k^{*} \partial y}$ (Magyari and Pantokratoras [41]), $\sigma^{*}$-Stephen-Boltzmann constant, the thermal field by Taylor's series, $T^{4}+3 T_{\infty}^{4}=4 T_{\infty}^{3} T$.

Dynamic similarity transformations is

$$
\begin{aligned}
& \psi=\sqrt{\frac{2 v_{f} U_{0}(x+b)^{n}}{(n+1)(x+b)^{-n}} f(\eta), \quad \eta=\sqrt{\frac{(n+1) U_{0}}{2 v_{f}(x+b)^{(1-n)}}} y,} \\
& \theta(\eta)=\frac{T-T_{\infty}}{T_{w}-T_{\infty}}, \phi(\eta)=\frac{\phi-\phi_{\infty}}{\phi_{w}-\phi_{\infty}} \\
& u(x+b)^{-n}=U_{0} f(\eta), \\
& v=-\sqrt{\frac{(n+1) v_{f} U_{0}(x+b)^{(n-1)}}{2}}\left(f(\eta)+\eta \frac{n-1}{n+1} f^{\prime}(\eta)\right)
\end{aligned}
$$

Flow function $\psi$-composed as $u=\frac{\partial \psi}{\partial y}, v=-\frac{\partial \psi}{\partial x}$ which sufficiently conduct the Eq. (1). Establish on the Eqs. $(7,8)$, the Eq. (1) is adequately formed whereas the Eqs. (2) and (6) become as

$$
f^{\prime \prime \prime}+G \cdot B\left(f f^{\prime \prime}-\frac{2 n}{n+1} f^{\prime 2}\right)+G \cdot A \cdot M\left(E_{1}-f^{\prime 2}\right)=0
$$

$\theta^{\prime \prime}+\operatorname{Pr} E \cdot\left(f \theta^{\prime}+(A 1) N b \theta^{\prime} \phi^{\prime}+(A 1) N t \theta^{\prime 2}\right.$

$$
\begin{aligned}
\left.+\left(\frac{1}{G . E}\right) E c \cdot f^{\prime \prime} 2++\frac{A \cdot M}{E} E c \cdot\left(f^{\prime}-E 1\right)^{2}\right) & =0 \\
\phi^{\prime \prime}+(A 1) \cdot\left(\frac{1}{(A 1)} \frac{N t}{N b} \theta^{\prime \prime}+\operatorname{Lef} \theta^{\prime}-\operatorname{Le\gamma } \phi\right) & =0
\end{aligned}
$$

with boundary conditions

$$
\begin{aligned}
& f(0)=\alpha \frac{(1-n)}{(1+n)}, f^{\prime}(0)=1, \theta(0)=1, \phi(0)=1 ; \\
& f^{\prime}(\infty)=1, \theta(\infty)=0, \phi(\infty)=0
\end{aligned}
$$




$$
\begin{aligned}
& \frac{K_{n f}}{\left(k_{s}+(e-1)\left(k_{f}\right)\right.}=1+\frac{(1-e) \zeta\left(k_{f}-\left(k_{s}\right)\right.}{\left(k_{s}+(e-1)\left(k_{f}\right)\right.}, \\
& K_{f}=\left(k_{s}+(e-1)\left(k_{f}\right)-\zeta\left(k_{f}-\left(k_{s}\right),\right.\right. \\
& B=\left(1-\zeta+\zeta \frac{\rho_{s}}{\rho_{f}}\right), T=\frac{K_{n f}}{K_{f}}, \\
& E=\left(1-\zeta+\zeta \frac{\rho_{s}(c p)_{s}}{\rho_{f}(c p)_{f}}\right) \text {, } \\
& E=\left(1-\zeta+\zeta \frac{\rho_{s}(c p)_{s}}{\rho_{f}(c p)_{f}}\right) \text {, } \\
& A=\left(\frac{3\left(\frac{\sigma_{s}}{\sigma_{f}}-1\right) \zeta}{\left(\frac{\sigma_{s}}{\sigma_{f}}+2\right)-\left(\frac{\sigma_{s}}{\sigma_{f}}-1\right) \zeta}+1\right), \\
& G=(1-\zeta)^{2.5}, A 1=\frac{1}{1-\zeta}
\end{aligned}
$$

$\operatorname{Pr}_{f}=\frac{\operatorname{Pr}}{\left(\frac{k_{n f}}{k_{f}}+\frac{4}{3} R a\right)}$-effective Prandtl number, $M=\frac{2 \sigma_{f} B_{0}^{2}}{c\left((n+1) \rho_{f}\right.}$ -magnetic parameter, $\gamma=\frac{2 k^{*}(x+b)^{(1-n)}}{U_{0}(n+1)}$ chemical reaction parameter, $R i=\frac{G r}{\operatorname{Re}_{x}^{2}}$ - Richardson number, $G r=\frac{g \beta\left(T_{w}-T_{\infty}\right) x^{3}}{v_{f}^{2}}$ -local Grashof number, $E_{1}=\frac{E_{0}(x+b)^{-n}}{B_{0} U_{0}}$ the electric parameter, $E C=\frac{U_{w}^{2}}{\left(c_{p}\right)_{f}\left(T_{w}-T_{\infty}\right)}$ the Eckert number, $R a=-\frac{4 \sigma^{*} T_{\infty}^{3}}{k_{f} k^{*}}$ the radiation-conduction parameter, $N_{b}=\frac{(\rho c)_{s}\left(D_{B}\right)_{f}\left(\phi_{w}-\phi_{\infty}\right)}{(\rho c)_{f} v_{f}}$ -Brownian motion and $N_{t}=\frac{(\rho c)_{s}\left(D_{T}\right)_{f}\left(T_{w}-T_{\infty}\right)}{(\rho c)_{f} v_{f} T_{\infty}}$-thermophoresis parameter. As well as the physical assets of engineering impacts of materials assisting utilizations are

$\operatorname{Re}_{x}^{\frac{1}{2}} C_{f}=\sqrt{\frac{n+1}{2}} \frac{f^{\prime \prime}(0)}{(1-\zeta)^{2.5}}$,

$\operatorname{Re}_{x}^{-\frac{1}{2}} N u_{x}=-\sqrt{\frac{n+1}{2}}\left(\frac{k_{n f}}{k_{f}}+\frac{4}{3} N\right)$

$\theta^{\prime}(0), S h_{x} \operatorname{Re}_{x}^{-\frac{1}{2}}=-\sqrt{\frac{n+1}{2}} \frac{\phi^{\prime}(0)}{(1-\zeta)}$

It is a fundamental law of thermodynamics that the Nusselt and Shear wood number can be negative, since it is defined as the dimensionless temperature and

Table 1 Thermal and physical asserts of fluid and particles

\begin{tabular}{lll}
\hline Thermophysical properties & $\mathrm{H}_{2} \mathrm{O}$ & AA7075 \\
\hline$\rho$ & 997.1 & 2810 \\
$c_{p}$ & 4179 & 960 \\
$k$ & 0.6129 & 173 \\
$\sigma$ & 0.05 & $26.77 \times 10^{6}$ \\
\hline
\end{tabular}

concentration gradient at the wall. The slope may be negative or positive, depending on the direction of heat flux. The skin friction coefficient based on the wall sheat stress magnitude has to be positive.

\section{Results and discussions}

In this scheme, nanoparticle shapes (sphere, cylinder and lamina) on MHD AA7075-water flow up a nonlinear devoted stretching from the cause of electric field in thermal radiation have been analyzed numerically. The system for Eqs. (9)-(11) are not be recognized exactly and algorithmic realization acceptable to the boundary conditions (12) are approved the very appreciable conducting software Maple 18. This software networks a fourth or fifth order Runge-Kutta Fehlberg technique with shooting

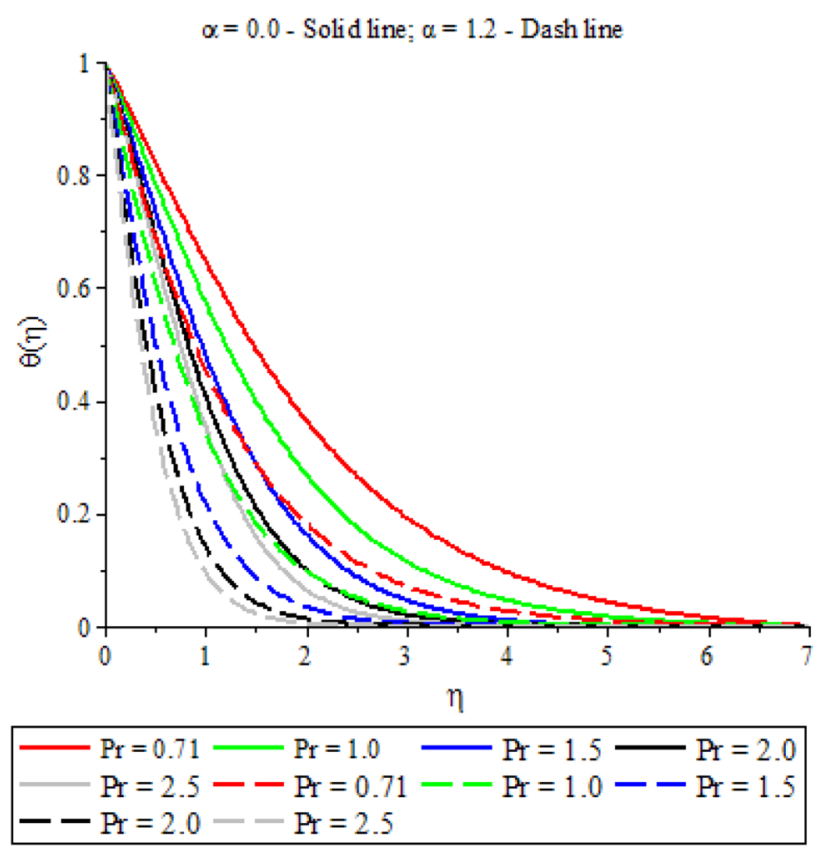

Fig. 2 Comparison of different Pr with Fig. 7 of Daniel et al. [43]

Table 2 Comparison of $-f^{\prime \prime}(0)$ when $E 1=M=0$

\begin{tabular}{lllll}
\hline Parameter & $\begin{array}{l}\text { Fang et al. } \\
\text { [42] }\end{array}$ & $\begin{array}{l}\text { Daniel et al. } \\
{[43]}\end{array}$ & Present result & Error \\
\hline$n, \alpha=0.25$ & & & & \\
10.0 & 1.1433 & 1.143316 & 1.143308 & $8.1 \mathrm{E}-06$ \\
9.0 & 1.1404 & 1.140388 & 1.140372 & $3.2 \mathrm{E}-06$ \\
7.0 & 1.1323 & 1.132281 & 1.132268 & $1.3 \mathrm{E}-06$ \\
5.0 & 1.1186 & 1.118587 & 1.118575 & $1.2 \mathrm{E}-06$ \\
3.0 & 1.0905 & 1.090490 & 1.090479 & $1.1 \mathrm{E}-06$ \\
1.0 & 1.0000 & 1.000001 & 1.000000 & $1.0 \mathrm{E}-06$ \\
\hline
\end{tabular}


Fig. 3 Temperature and concentration profiles for Magnetic field
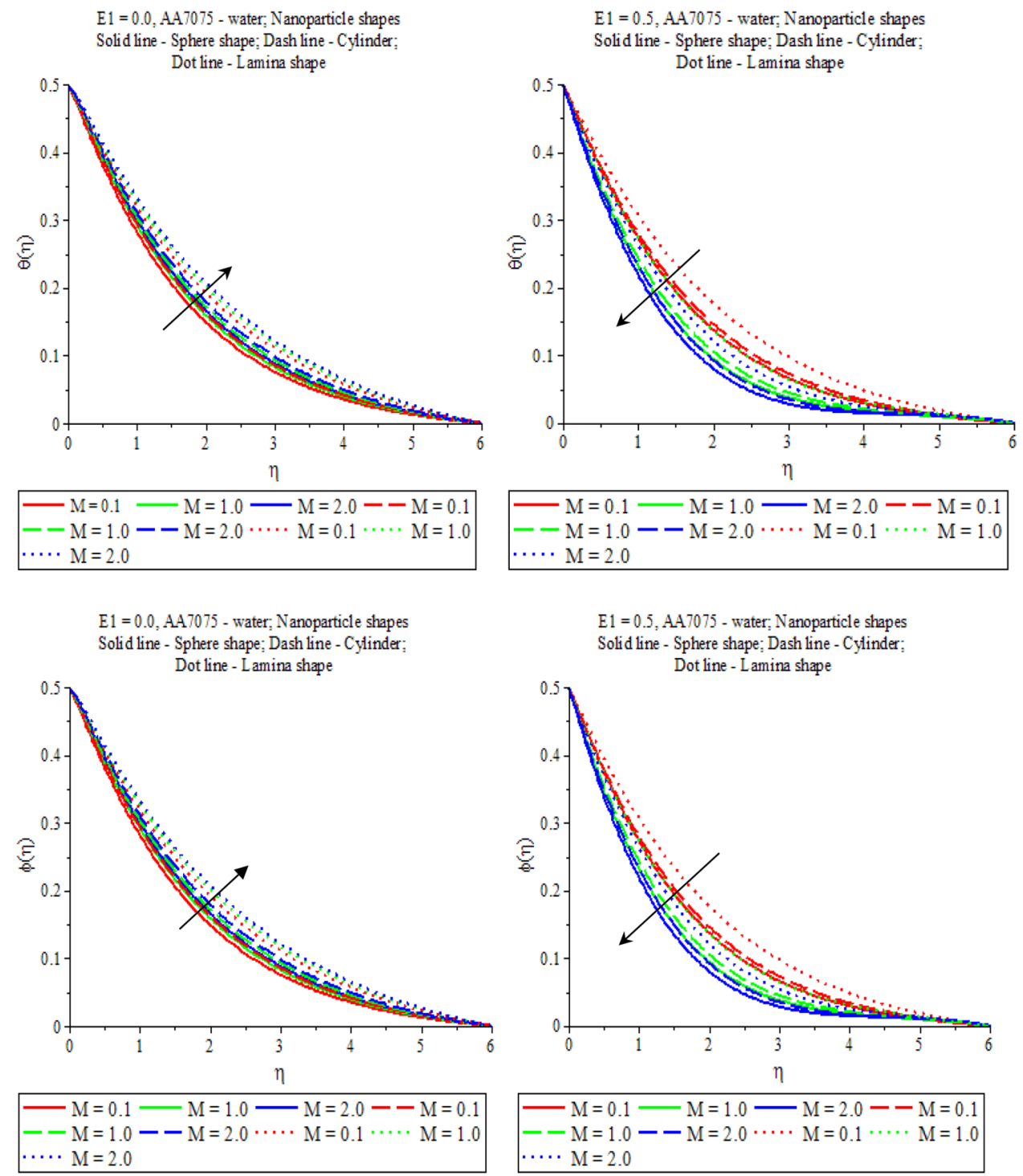

system as persistent to execute the boundary value problems numerically proceeding the classification model. The Prandtl number range of the nanofluid is assumed as $6.2 \leq \operatorname{Pr} \leq 10.4$.

\subsection{Affirmation}

Analytical formation is to observe the pattern and realistic events raised the classic issue and confirm the numerical scheme. The numerical application is highly to decode confusion, physically or geometrically. We admit absolutely algorithm control numerical software automatically to appreciable engrossment. In the exploration system, most of the investigator doesn't constitute numerical pattern to investigate the problem fasts from enhancement construction. But we ought to transit in mind that all the software, precisely system has been accepting the analytical fulfillment lately. Analytical structures are employed to initial problem. However, the numerical schemes are excited for empirical aggregation (Table 1).

It is accepted of the Fig. 2 and Table 2, theoretical perception of $\theta(\eta)$ and $-f^{\prime \prime}(0)$ for noticeable values of the parameter are associated with Fig. 7 and Table 1 of Daniel et al. [43] respectively.

\subsection{Magnetic effects on temperature and concentration profiles, Fig. 3}

In the presence of electric field $E_{1}=0 . .5$, it is observed that the temperature and concentration of AA7075-water nanofluid decrease with increase of magnetic strength. In the presence of $E_{1}=0.0$, it is seen that the temperature 
Fig. 4 Temperature and concentration profiles for thermal radiation
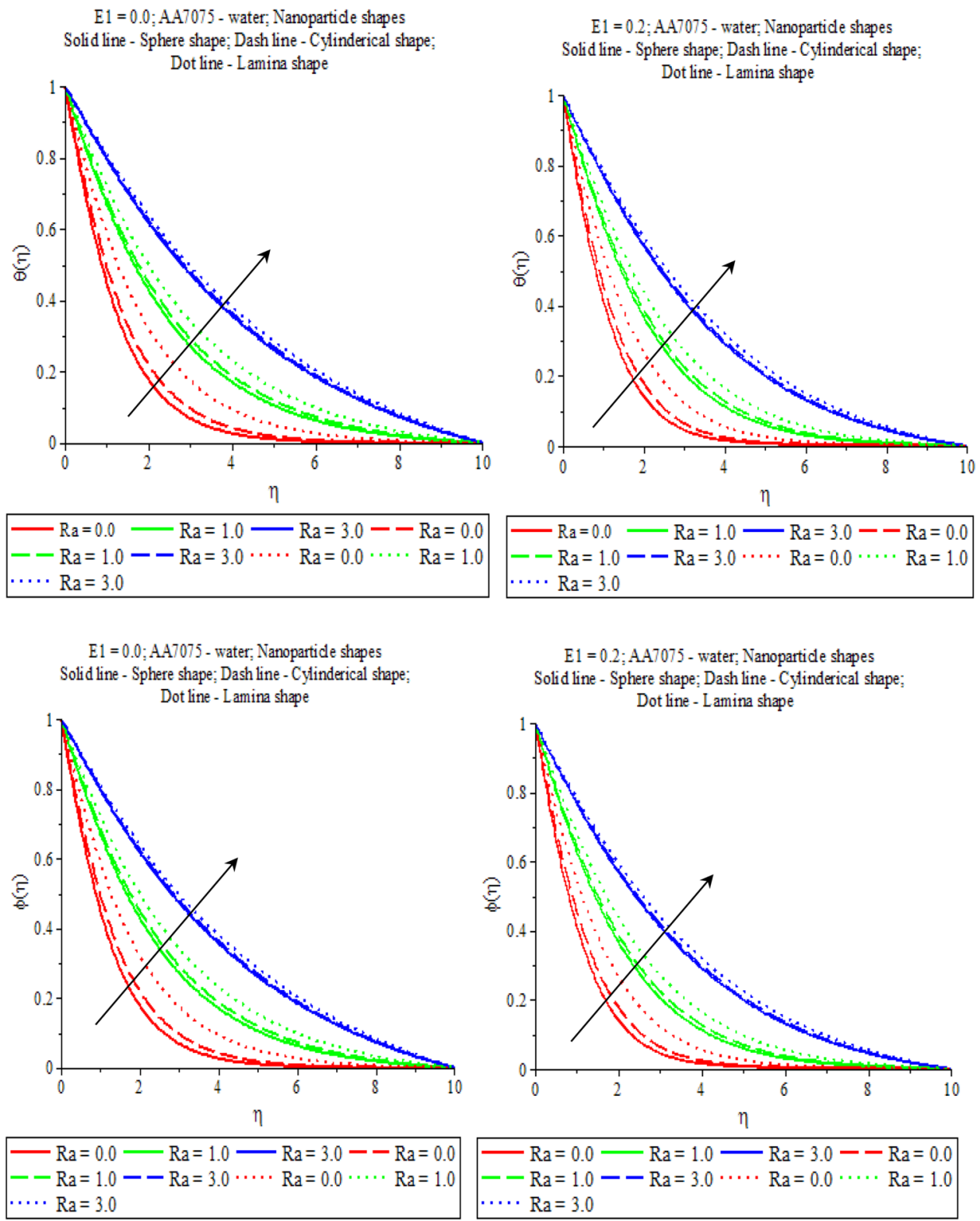

and concentration of AA7075-water increase with increase of magnetic strength. In the presence of electric field, thermal and diffusive boundary layer thickness of lamina shape particles on AA7075-water is stronger as compared to other shapes in the flow regime. This is due to the evidence that the magnetic lines of forces move over the plate and the fluid which is decreased by the viscous force, obtains an effort from the magnetic field strength which prevents the viscous effects. Hence the temperature and concentration of the AA7075-water decelerate as the parameter $M$ increases in the presence of electric field, Fig. 3.

\subsection{Thermal radiation energy effects on temperature and concentration profiles,} Fig. 4

In the presence of electric field, it is predicted that the temperature and concentration of AA7075-water nanofluid increase with increase of thermal radiation energy strength. In the presence of electric field, thermal and diffusive boundary layer stiffness of lamina shape particles on AA7075-water is more significant as compared to other shapes in the flow regime because of low level thermal conductivity of base fluid (water), Table 1. The increase in thermal radiation energy aids the commute of heat energy from the flow region and so the fluid temperature decelerates. A decelerate in the ranges of forgiven and 
Fig. 5 Temperature and concentration for non-linear wall strength
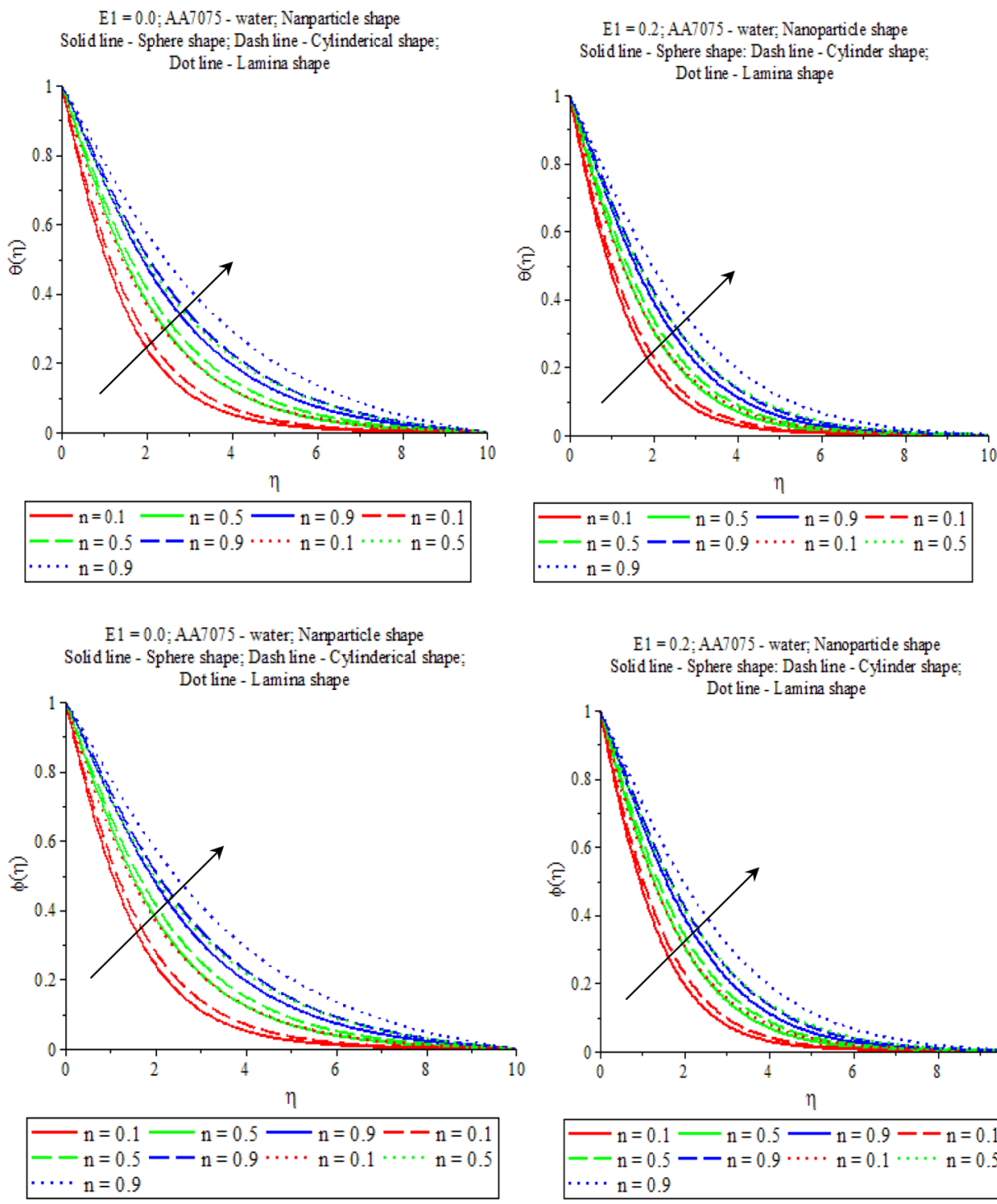

aids a reduce in the Rosseland radiation absorptivity. Since divergence of the radiative heat flux enhances, decelerates which in turn roots to enhance the rate of radiative heat transfer to the AA7075-water and hence the nanofluid temperature and concentration increase. This means that the thermal boundary layer decelerates and more uniform temperature and concentration distribution across the boundary layer.

\subsection{Non-linear wall strength on temperature and concentration profiles, Fig. 5}

In the presence of $\mathrm{E} 1=0.0$ and $\mathrm{E} 1=0.2$, it is noticed that the temperature and concentration of AA7075-water nanofluid enhance with increase of non-linear wall strength. In the existence of electric field, thermal and diffusive boundary layer stiffness of lamina shape particles on AA7075-water is higher as compared to other shapes in the flow regime.

\subsection{Nanoparticle volume fraction on temperature and concentration profiles, Fig. 6}

In the presence of $\mathrm{E} 1=0.0$ and $\mathrm{E} 1=0.2$, the temperature and concentration of AA7075-water accelerate as nanoparticle volume fraction strength enlarges. It is noted that the thermal and diffusive boundary layer thickness of lamina shape particles on AA7075-water flow is powerful as correlated to other shapes in the flow regime. This is as a result of AA7075 has immense thermal conductivity and its inclusion to the water enhances the thermal conductivity for the nanofluid, so the stiffness of the thermal boundary layer raises. It is also noticed that with rising the volume fraction of the nanoparticles, the thermal 
Fig. 6 Temperature and concentration nanoparticle volume fraction
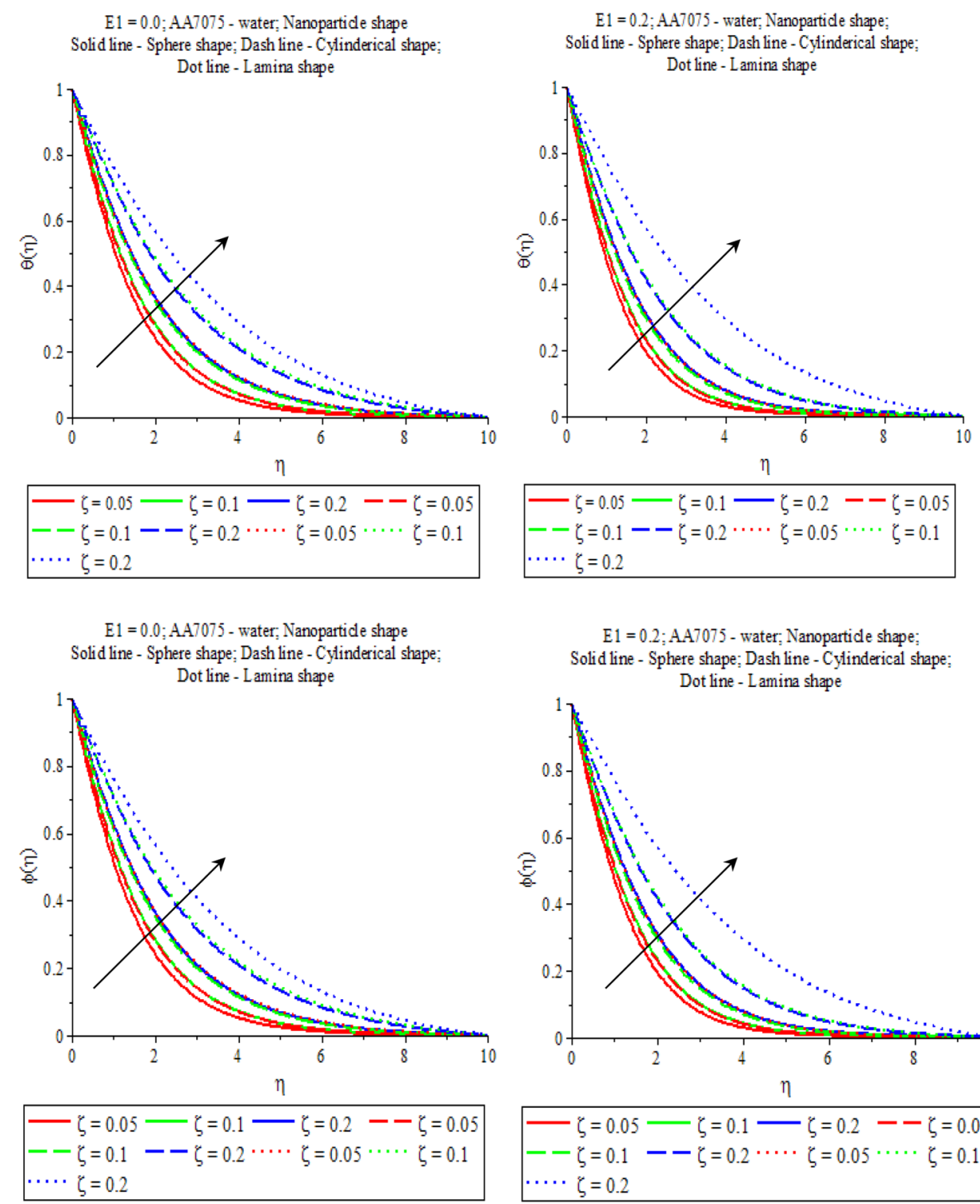

boundary layer is enhanced. This admits with the physical attitude of nanoparticles. This information predicts that the cause of nanofluids will be implication in the cooling and heating movements.

\subsection{Thermophoresis particle deposition on temperature and concentration profiles,} Fig. 7

Thermophoresis is a phenomenon noticed in mixtures of mobile nanoparticles where the various particle types display different responses to the force of a temperature gradient, Abdel-Wahed et al. [44]. Justify this phenomenon appears in this study through the thermophoresis parameter Nt such that increasing this parameter leads to increasing of boundary layer temperature and nanoparticle concentration as shown in Fig. 7. Both $E 1=0.0$ and $\mathrm{E} 1=0.5$, it is shown that the temperature and concentration on AA7075-water accelerate with increase of thermophoresis particle deposition. Thermal and diffusive boundary layer thickness of lamina shaped particles of AA7075-water in the presence of electric field, E1 $=0.5$ is more efficient as associated with $E 1=0.0$. This is due to the combined effect of Brownian and thermophoresis diffusion coefficient strength in the presence of kinematic viscosity of AA7075-water. 
Fig. 7 Thermophoresis on temperature and concentration with or without electrical field
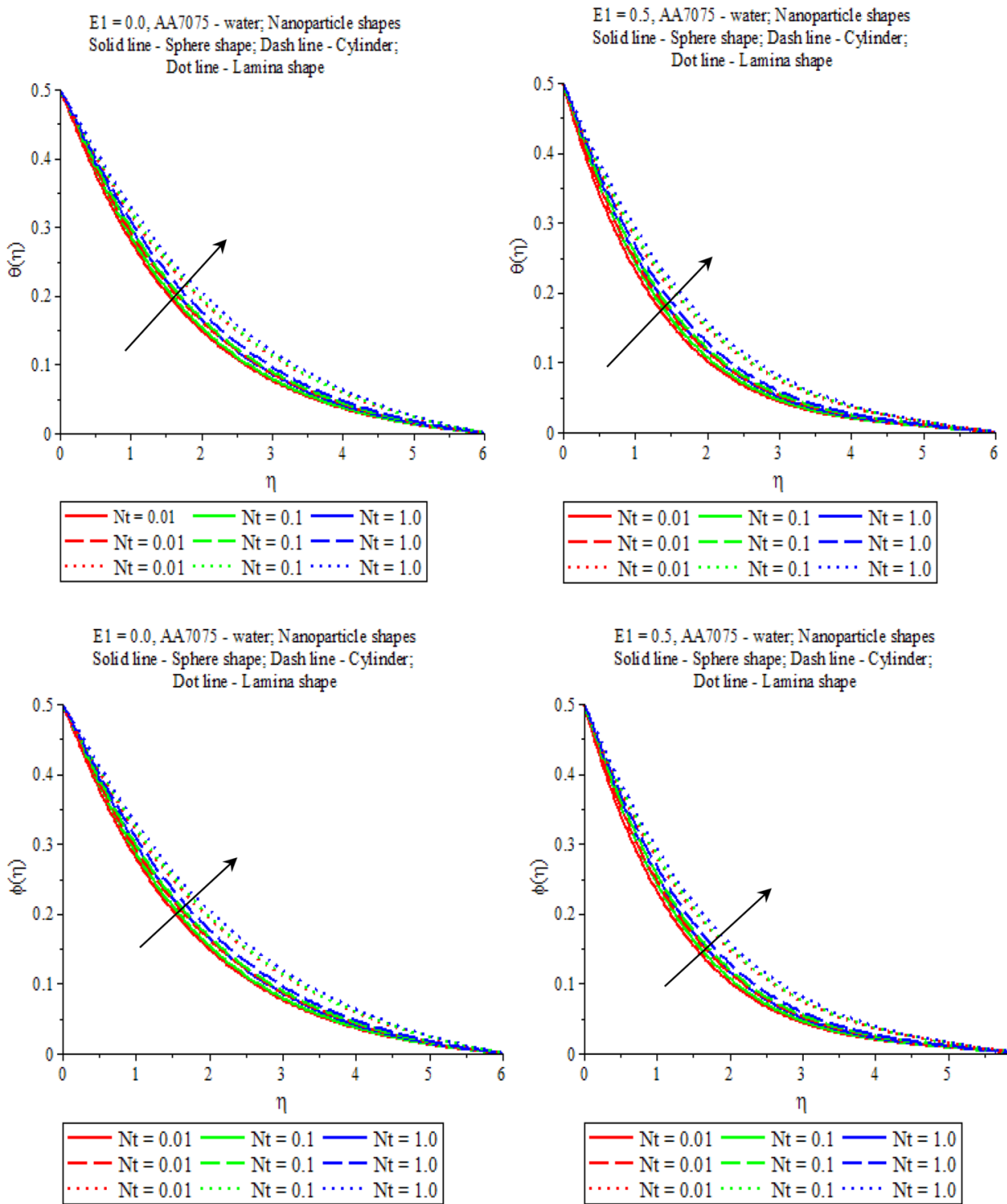

Table 3 Nanoparticle volume fraction and shape on $f^{\prime \prime}(0),-\theta^{\prime}(0)$ and $-\phi^{\prime}(0)$ with or without electric field $(E 1=0.2)$ in the presence of AA7075-water

\begin{tabular}{lllll}
\hline$\zeta$ & $f^{\prime \prime}(0)$ & $-\theta^{\prime}(0)$ & $-\phi^{\prime}(0)$ & AA7075 shapes \\
\hline 0.05 & -1.572663 & $\mathbf{0 . 5 2 1 3 0 0}$ & 2.408874 & Sphere shape \\
0.1 & -1.511998 & 0.460948 & 2.534099 & \\
0.2 & -1.361190 & 0.360827 & 2.817025 & \\
0.05 & -1.572663 & 0.474963 & 2.423611 & Cylindrical shape \\
0.1 & -1.511998 & 0.390063 & 2.555679 & \\
0.2 & -1.361190 & 0.276281 & $\mathbf{2 . 8 3 9 9 7 0}$ & \\
0.05 & -1.572663 & 0.383708 & $\mathbf{2 . 4 5 0 5 2 1}$ & Lamina shape \\
0.1 & -1.511998 & 0.281016 & 2.584528 & \\
0.2 & -1.361190 & $\mathbf{0 . 2 2 6 8 6 1}$ & 2.838250 & \\
\hline
\end{tabular}

\subsection{Nanoparticle volume fraction on skin friction, rate of heat and mass transfer, Table 3}

In the presence of $E 1=0.2$, heat/mass transfer rate of AA7075-water decreases/increases with increase of $\zeta$. The rate of heat/mass transfer of sphere/cylindrical shaped nanoparticles on AA7075-water is stronger at $\left(\zeta=0.05,-\theta^{\prime}(0)=0.521300 /\left(\zeta=0.2,-\phi^{\prime}(0)=2.839970\right.\right.$ whereas the heat/mass transfer rate of lamina shaped nanoparticles on AA7075-water at $\left(\zeta=0.2,-\theta^{\prime}(0)=0.226861\right.$ $/\left(\zeta=0.05,-\phi^{\prime}(\eta)=2.450521\right.$ is weaker as related to other particle shapes in the flow regime with increase of $\zeta$. 


\section{Conclusion}

Impact of electric field strength on AA7075-water convective flow through a non-linear stretching wall is presented using Runge-Kutta Felhberg method with shooting technique, MAPLE 18. Effects of magnetic strength, thermal radiation energy, thermophoresis particle deposition, particle shapes and volume fraction on AA7075-water nanofluid behaviour are taken into account in the subsistence of electric field. It is interesting to note that the thermal and diffusive thermal boundary layer thickness of lamina shaped AA7075-water, $E_{1}=0.2$ is more significant as compared to $E_{1}=0.0$ because of the combined effects of electric and magnetic strength with increase of all parameters strength. In the presence of electric field, it is noted that the temperature and concentration of AA7075water increase with increase of all parameters strength while it decreases with rise of magnetic strength. Heat/ mass transfer rate of sphere/cylindrical shaped particles on AA7075-water is stronger whereas the lamina shaped particles on AA7075-water is weaker as related to other shapes with rise of nanoparticle volume fraction.

Acknowledgement Authors thanks to UTHM and Higher Education Ministry, Malaysia for their monetary assistance, (Geran Penyelidikan Kontrak/U687/2016-2018).

\section{Compliance with ethical standards}

Conflict of interest The authors declare that they have no conflict of interest.

\section{References}

1. Yoshio Y (2015) Extra super duralumin and successive aluminum alloys for aircraft. J Jpn Inst Light Met 39:379

2. Sandeep N, Animasaun IL (2017) Heat transfer in wall jet flow of magnetic-nanofluids with variable magnetic field. Alex Eng J 56:263-269

3. Sandeep N, Sharma RP, Ferdows M (2017) Enhanced heat transfer in unsteady magnetohydrodynamic nanofluid flow embedded with aluminum alloy nanoparticles. J Mol Liq 56:263-269. https://doi.org/10.1016/j.molliq.2017.03.051

4. Sandeep N, Animasaun IL (2017) Heat transfer in wall jet flow of magnetic-nanofluids with variable magnetic field. Alex Eng J 56(2):263-269. https://doi.org/10.1016/j.aej.2016.12.019

5. Makinde OD, Animasaun IL (2016) Bioconvection in MHD nanofluid flow with nonlinear thermal radiation and quartic autocatalysis chemical reaction past an upper surface of a paraboloid of revolution. Int J Therm Sci 109:159-171

6. Makinde OD, Animasaun IL (2016) Thermophoresis and Brownian motion effects on MHD bioconvection of nanofluid with nonlinear thermal radiation and quartic chemical reaction past an upper horizontal surface of a paraboloid of revolution. J Mol Liq 221:733-743

7. Animasaun IL, Kọrikọ OK, Adegbie KS, Mahanthesh B (2018) Comparative analysis between $36 \mathrm{~nm}$ and $47 \mathrm{~nm}$ alumina-water nanofluid flows in the presence of Hall effect. J Therm Anal Calorim. https://doi.org/10.1007/s10973-018-7379-4

8. Selimefendigil F, Oztop HF (2016) Conjugate natural convection in a cavity with a conductive partition and filled with different nanofluids on different sides of the partition. J Mol Liq 216:67-77

9. Abramenko N, Marukovich El, Anisovich AG (2013) Heat transfer in alloys. Surf Eng Appl Electrochem 49:480-484

10. Huray PG (2009) Maxwell's equations. Wiley, Hoboken, p 22. ISBN 0-470-54276-4

11. Dahl PF (1997) Flash of the cathode rays: a history of J J Thomson's electron. CRC Press, Boca Raton, $\mathrm{p} 10$

12. Muhaimin I, Kandasamy R, Hashim I (2009) Thermophoresis and chemical reaction effects on non-Darcy MHD mixed convective heat and mass transfer past a porous wedge in the presence of variable stream condition. Chem Eng Res Des 87(11):1527-1535

13. Bachok N, Ishak A, Nazar R, Senu N (2013) Stagnation-point flow over a permeable stretching/shrinking sheet in a copper-water nanofluid. Bound Value Probl 39:1-10

14. Rana P, Bhargava R (2012) Flow and heat and transfer of a nanofluid over a nonlinearly stretching sheet: a numerical study. Commun Nonlinear Sci Numer Simul 17:212-226

15. Choi SUS (1995) Enhancing thermal conductivity of fluids with nanoparticles. ASME Publ Fed 231:99-106

16. Lin Y, Zheng L, Zhang X, Ma L, Chen G (2015) MHD pseudo-plastic nanofluid unsteady flow and heat transfer in a finite thin film over stretching surface with internal heat generation. Int J Heat Mass Transf 84:903-911

17. Zhang C, Zheng L, Zhang X, Chen G (2015) MHD flow and radiation heat transfer of nanofluids in porous media with variable surface heat flux and chemical reaction. Appl Math Model 39(1):165-181

18. Lin Y, Zheng L, Zhang X (2014) Radiation effects on Marangoni convection flow and heat transfer in pseudo-plastic non-Newtonian nanofluids with variable thermal conductivity. Int J Heat Mass Transf 77:708-716

19. Zheng $L$, Zhang C, Zhang $X$, Zhang J (2013) Flow and radiation heat transfer of a nanofluid over a stretching sheet with velocity slip and temperature jump in porous medium. J Frankl Inst 350(5):990-1007

20. Li Z, Khan I, Shafee A, Tlili I, Asifa T (2018) Energy transfer of Jeffery-Hamel nanofluid flow between non-parallel walls using Maxwell-Garnetts (MG) and Brinkman models. Energy Rep 4:393-399

21. Afridi MI, Qasim M, Khan I, Tlili I (2018) Entropy generation in MHD mixed convection stagnation-point flow in the presence of joule and frictional heating. Case Stud Therm Eng 12:292-300

22. Khalid A, Khan I, Khan A, Shafie S, Tlili I (2018) Case study of MHD blood flow in a porous medium with CNTS and thermal analysis. Case Stud Therm Eng 12:374-380

23. Khan I, Abro KA, Mirbhar MN, Tlili I (2018) Thermal analysis in Stokes' second problem of nanofluid: applications in thermal engineering. Case Stud Therm Eng 12:271-275

24. Abro KA, Rashidi MM, Khan I, Abro IA, Tassaddiq A (2018) Analysis of Stokes' second problem for nanofluids using modern approach of Atangana-Baleanu fractional derivative. J Nanofluids 7:738-747

25. Aman S, Khan I, Ismail Z, Salleh MZ (2018) Impacts of gold nanoparticles on MHD mixed convection Poiseuille flow of nanofluid passing through a porous medium in the presence of thermal radiation, thermal diffusion and chemical reaction. Neural Comput Appl 30(3):789-797

26. Khan Ml, Hayat T, Khan Ml, Alsaedi A (2017) A modified homogeneous-heterogeneous reactions for MHD stagnation flow with viscous dissipation and Joule heating. Int J Heat Mass Transf 113:310-317 
27. Hayat T, Khan MI, Farooq M, Yasmeen T, Alsaedi A (2016) Stagnation point flow with Cattaneo-Christov heat flux and homogeneous-heterogeneous reactions. J Mol Liq 220:49-55

28. Khan MI, Hayat T, Waqas M, Khan MI, Alsaedi A (2017) Impact of heat generation/absorption and homogeneous-heterogeneous reactions on flow of Maxwell fluid. J Mol Liq 233:465-470

29. Daniel YS, Daniel SK (2015) Effects of buoyancy and thermal radiation on MHD flow over a stretching porous sheet using homotopy analysis method. Alex Eng J 54(3):705-712

30. Khan M-I, Hayat T, Waqas M, Alsaedi A (2017) Outcome for chemically reactive aspect in flow of tangent hyperbolic material. $J$ Mol Liq 230:143-151

31. Daniel YS (2016) Laminar convective boundary layer slip flow over a flat plate using homotopy analysis method. J Inst Eng (India) Ser E 97(2):115-121

32. Khan A, Khan D, Khan I, Ali F, ul Karim F, Imran M (2018) MHD flow of sodium alginate-based casson type nanofluid passing through a porous medium with newtonian heating. Sci Rep 8(1):8645

33. Aman S, Khan I, Ismail Z, Salleh MZ, Tlili I (2018) A new Caputo time fractional model for heat transfer enhancement of water based graphene nanofluid: an application to solar energy. Results Phys 9:1352-1362

34. Gul A, Khan I, Makhanov SS (2018) Entropy generation in a mixed convection Poiseulle flow of molybdenum disulphide Jeffrey nanofluid. Results Phys 9:947-954

35. Hussanan A, Salleh MZ, Khan I (2018) Microstructure and inertial characteristics of a magnetite ferrofluid over a stretching/shrinking sheet using effective thermal conductivity model. J Mol Liq 255:64-75

36. Ahmed TN, Khan I (2018) Mixed convection flow of sodium alginate (SA-NaAlg) based molybdenum disulphide (MoS2) nanofluids: Maxwell Garnetts and Brinkman models. Results Phys 8:752-757

37. Tlili I, Khan WA, Khan I (2018) Multiple slips effects on MHD SA$\mathrm{Al}_{2} \mathrm{O}_{3}$ and $\mathrm{SA}-\mathrm{Cu}$ non-Newtonian nanofluids flow over a stretching cylinder in porous medium with radiation and chemical reaction. Results Phys 8:213-222

38. Saqib M, Ali F, Khan I, Sheikh NA, Khan A (2018) Entropy generation in different types of fractionalized nanofluids. Arab J Sci Eng. https://doi.org/10.1007/s13369-018-3342-8

39. Suresh S, Venkitaraj KP, Hameed MS, Sarangan J (2014) Turbulent heat transfer and pressure drop characteristics of dilute water based $\mathrm{Al}_{2} \mathrm{O}_{3} \mathrm{Cu}$ hybrid nanofluids. J Nanosci Nanotechnol $14: 25632572$

40. Maxwell JCA (1891) A treatise on electricity and magnetism, 2 unabridged, 3rd edn. Clarendon Press, Oxford

41. Magyari E, Pantokratoras A (2011) Note on the effect of thermal radiation in the linearized Rosseland approximation on the heat transfer characteristic of various boundary layer flow. Int Commun Heat Mass Transf 38:554-556

42. Fang $T$, Zhang J, Zhong $Y$ (2012) Boundary layer flow over a stretching sheet with variable thickness. Appl Math Comput 218(13):7241-7252

43. Daniel YS, Aziz ZA, Ismail Z, Salah F (2017) Impact of thermal radiation on electrical MHD flow of nanofluid over nonlinear stretching sheet with variable thickness. Alex Eng J. https://doi. org/10.1016/j.aej.2017.07.007

44. Abdel-Wahed MS, Elbashbeshy EMA, Emam TG (2015) Flow and heat transfer over a moving surface with non-linear velocity and variable thickness in a nanofluids in the presence of Brownian motion. Appl Math Comput 254:49-62 